\title{
Comparing the tensile strength of square and reversing half-hitch alternating post knots
}

\author{
Vincent $\mathrm{Wu}, \mathrm{BHSc}$ \\ Edward A. Sykes, PhD \\ Dale Mercer, MD \\ Wilma M. Hopman, MA \\ Ephraim Tang, MD, MSc
}

Accepted Nov. 10, 2016; Early-released

Apr. 1, 2017

\section{Correspondence to:}

E. Tang

Department of Surgery, Victory 3

Kingston General Hospital

76 Stuart St

Kingston ON K7L 2V7

tange1@kgh.kari.net

DOI: $10.1503 /$ cjs.009716
Background: Square knots are the gold standard in hand-tie wound closure, but are difficult to reproduce in deep cavities, inadvertently resulting in slipknots. The reversing half-hitch alternating post (RHAP) knot has been suggested as an alternative owing to its nonslip nature and reproducibility in limited spaces. We explored whether the RHAP knot is noninferior to the square knot by assessing tensile strength.

Methods: We conducted 10 trials for each baseline and knot configuration, using 3-0 silk and 3-0 polyglactin 910 sutures. We compared tensile strength between knot configurations at the point of knot failure between slippage and breakage.

Results: Maximal failure strength (mean \pm SD) in square knots was reached with 4-throw in both silk $(30 \pm 1.5 \mathrm{~N})$ and polyglactin $910(39 \pm 12 \mathrm{~N})$. For RHAP knots, maximal failure strength was reached at 5 -throw for both silk $(31 \pm 1.5 \mathrm{~N})$ and polyglactin $910(41 \pm 13 \mathrm{~N})$. In both sutures, there were no strength differences between 3-throw square and 4-throw RHAP, between 4-throw square and 5-throw RHAP, or between 5-throw square and 6-throw RHAP knots. Polyglactin 910 sutures, in all knot configurations, were more prone to slippage than silk sutures $(p<0.001)$.

Conclusion: The difference in mean tensile strength could be attributed to the proportion of knot slippage versus breakage, which is material-dependent. Future studies can re-evaluate findings in monofilament sutures and objectively assess the reproducibility of square and RHAP knots in deep cavities. Our results indicate that RHAP knots composed of 1 extra throw provide equivalent strength to square knots and may be an alternative when performing hand-ties in limited cavities with either silk or polyglactin 910 sutures.

Contexte : Les nœuds plats sont la norme en matière de points de suture manuels, mais ils sont difficiles à reproduire dans des cavités profondes et deviennent par inadvertance des nœuds coulants. Le nœud de type demi-clé inversée alternée a été proposé comme solution de rechange en raison de sa nature non glissante et de sa reproductibilité dans des espaces restreints. Nous avons voulu vérifier la noninfériorité de la demi-clé inversée alternée par rapport au nœud plat en évaluant sa résistance à la traction.

Méthodes : Nous avons réalisé 10 essais de référence et autant d'essais pour chaque type de nœud, en utilisant des fils de soie de taille 3-0 et de polyglactine 910 de taille 3-0. Nous avons comparé la résistance à la traction des différentes configurations de nœuds, c'est-à-dire le point de défaillance entre le glissement du nœud et la rupture de celui-ci.

Résultats : La résistance maximale à la traction (moyenne \pm É.-T.) des nœuds plats a été atteinte avec 4 boucles, tant pour le fil de soie $(30 \pm 1,5 \mathrm{~N})$ que le fil de polyglactine $910(39 \pm 12 \mathrm{~N})$. Pour les demi-clés inversées alternées, la résistance maximale à la traction a été atteinte avec 5 boucles pour le fil de soie $(31 \pm 1,5 \mathrm{~N})$ et de polyglactine $910(41 \pm 13 \mathrm{~N})$. Dans les 2 types de sutures, on n'a noté aucune différence de résistance entre le nœud plat à 3 boucles et la demi-clé inversée alternée à 4 boucles, entre le nœud plat à 4 boucles et la demi-clé inversée alternée à 5 boucles, ni entre le nœud plat à 5 boucles et la demi-clé inversée alternée à 6 boucles. Dans tous les types de nœuds, les sutures de polyglactine 910 ont été plus sujettes au glissement que les sutures de soie $(p<0,001)$. 


\begin{abstract}
Conclusion : La différence de résistance moyenne à la traction a pu être attribuée à la proportion de glissement c. la rupture des nœuds, qui dépend du matériau. D'autres études pourraient réévaluer les résultats obtenus avec des sutures monofilaments et mesurer objectivement la reproductibilité des nœuds plats et des demi-clés inversées alternées dans des cavités profondes. Selon nos résultats, les demi-clés inversées alternées comportant une boucle supplémentaire offrent une résistance équivalente à celle des nœuds plats et seraient une solution de rechange lors de sutures manuelles dans des cavités restreintes, avec la soie ou la polyglactine 910 .
\end{abstract}

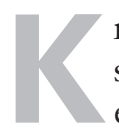

not tying is a basic surgical technique that allows sutures and ligatures to resist physiologic wound expansion during the normal healing process. ${ }^{1,2}$ Failure of surgical knots can lead to complications postsurgery, especially for internal repairs within deep body cavities that cannot be readily visualized under direct observation. ${ }^{3}$

The mechanism of knot failure is divided into slippage and breakage. When referring to the knot configurations, a throw denotes the weaving of the suture strands, with a knot consisting of 2 or more throws in succession. If an inadequate number of throws are used in the knot construction, the main mechanism of failure is by slippage or unravelling of the knot. The degree of slippage depends on a variety of factors, including knot type, suture material, suture gauge and moisture. With additional throws, increased friction is created between the suture materials, transitioning the mechanism of knot failure from slippage to breakage. The point where the suture breaks is most often at the knot, with the force required to break a knot lower than that of the untied suture material. ${ }^{4}$ Increasing the number of throws during knot construction increases knot strength, but only up to the point where the mechanism of failure transitions from slippage to breakage. When all knots fail by breakage, additional throws will not provide increased tensile strength. ${ }^{5}$

The square knot is a type of reef knot with a singlelooped overhand throw, followed by a reversed overhand throw laid flat on top. Its counterpart, the surgeon's knot, is a double-looped first-throw variation. Both have been used for nearly a century in wound repair and closure. ${ }^{6,7}$ Today, the square knot is used as the gold standard for freehand knot tying in surgery, as it is the most secure especially when there is risk of tearing through delicate tissue. ${ }^{10}$ However, because of the flat nature of the square knot, it is difficult to reproduce during surgery, as tension must be applied equally to both ends of the strand perpendicular to the axis of the knot. This is especially the case when tying a square knot within the limited space of a deep body cavity, where throws inadvertently not laid parallel to one another, often owing to greater tension applied to one strand of the suture, can create a slipknot. ${ }^{10}$ This is also known as the nonalternating post knot, which is generally weaker and has a greater chance of failure, as it is more prone to slippage. ${ }^{1,2,10}$

The reversing half-hitch alternating post (RHAP) knot is an alternative to the square knot that addresses the issue of slippage seen in nonalternating post knots. The RHAP knot alternates posts with each successive throw to prevent the knot from sliding off of a single standing suture strand. The RHAP knot is also postulated to be easier to tie than the square knot within a deep cavity. Although it has been suggested that square knots and RHAP knots with 1 extra throw have similar strengths and knot security, quantitative comparisons of the strength of the square knot versus the RHAP knot have yet to be established. ${ }^{11}$

In the present study we sought to assess whether the RHAP knot with an additional throw could impart equivalent strength to the square knot by examining the tensile breaking strengths and mode of failures of each using an array of suture materials.

\section{Methods}

\section{Materials}

Experiments were conducted in the Biomedical Laboratory at the Human Mobility Research Centre, Kingston General Hospital, Ontario, Canada. The ElectroForce 5500 Static Pull Machine (Bose Corporation) was used to measure the critical tensile breaking strength of each suture knot. We tested the RHAP and square knots with 3-0 gauged silk (PERMA-SILKÒ, Ethicon), polyglactin 910 (VICRYLÒ, Ethicon), polypropylene (PROLENEÒ, Ethicon) and polydioxanone (PDSO II, Ethicon) sutures. However, polypropylene and polydioxanone monofilament sutures underwent plastic deformation and irreversible stretching of the suture material and did not fail by breakage during baseline or knot configuration testing. Thus, we excluded monofilaments from the final data analysis.

\section{Experimental setup}

Adapted from the experimental setup used by Trimbos ${ }^{12}$ to construct knotted suture loops, we tied suture knots around 2 cylindrical rods attached on a board to ensure reproducibility of the length of each loop. The rods were removable and allowed the sutures to be transferred to the static pull machine for assessment of knot strength. Knot failure by slippage was defined as $3 \mathrm{~mm}$ of loop elongation, and this criterion has been widely used in the evaluation of various suture and knot combinations. ${ }^{1-5,8-10,13-16}$ One of us (V.W.) tied all knots to ensure consistency, as this was a commonly accepted method for knot construction across the literature on knot biomechanics. ${ }^{8,12-15}$ 
Surgical loops were attached to the device via 2 mounting arms, with the suture knot free-hanging. Once properly positioned, the surgical loop was pretensioned by allowing the lower mounting arm to extend by the force of gravity and by fixing the lower mounting arm to its hanging length (Fig. 1). We assessed tensile loading by configuring the static pull machine to separate the mounting arms at a constant rate of $1 \mathrm{~mm} / \mathrm{s}$ up to the maximal displacement of $10 \mathrm{~mm}$. Ten trials were conducted for each knot configuration in each suture material. This sample size was based on previous studies of suture knot breaking strength. Mahar and colleagues ${ }^{13}$ were able to demonstrate a significant difference in the breaking strength of 3 knot configurations within 2 suture materials.

\section{Baseline testing}

Initial baseline testing was used to assess the inherent tensile strength of each suture material. A single strand of unknotted, dry suture was mounted onto the experimental setup by placing the suture material around the upper mounting arm and then looping each free end of the strand 3 times around the lower mounting arm, securing the loose ends in place with stainless steel self-locking forceps, thereby creating an unknotted loop of suture. Tensile forces on the suture material were automatically recorded from the static pull machine in Newtons. We defined the baseline tensile strength for each suture as the maximum force where the suture material either failed or stretched beyond $10 \mathrm{~mm}$. We conducted 10 trials for each suture material to establish the baseline tensile strength.

\section{Square knot testing}

Surgical loops of each suture material (silk and polyglactin 910) were constructed under dry conditions, with square knots composed of 3-5 throws using standard surgical knot tying techniques. ${ }^{6}$ Using the commonly accepted knot nomenclature by Loutzenheiser and colleauges, ${ }^{15}$ the configuration of a 4-throw square knot was $1=1=1=1$ (Fig. 2). These suture loops were then mounted onto the static pull machine to examine the association between the number of square knot throws and knot strength. We defined knot strength as the maximum force exhibited onto the surgical loop before knot breakage or knot slippage (exceeding $3 \mathrm{~mm}$ ). Force measurements were automatically recorded by the static pull machine in Newtons. We conducted 10 trials for each suture material and square knot.

\section{RHAP knot testing}

The name of the RHAP knot denotes its construction and consists of 2 components. First, half-hitch throws alternate between forehand and backhand variations.

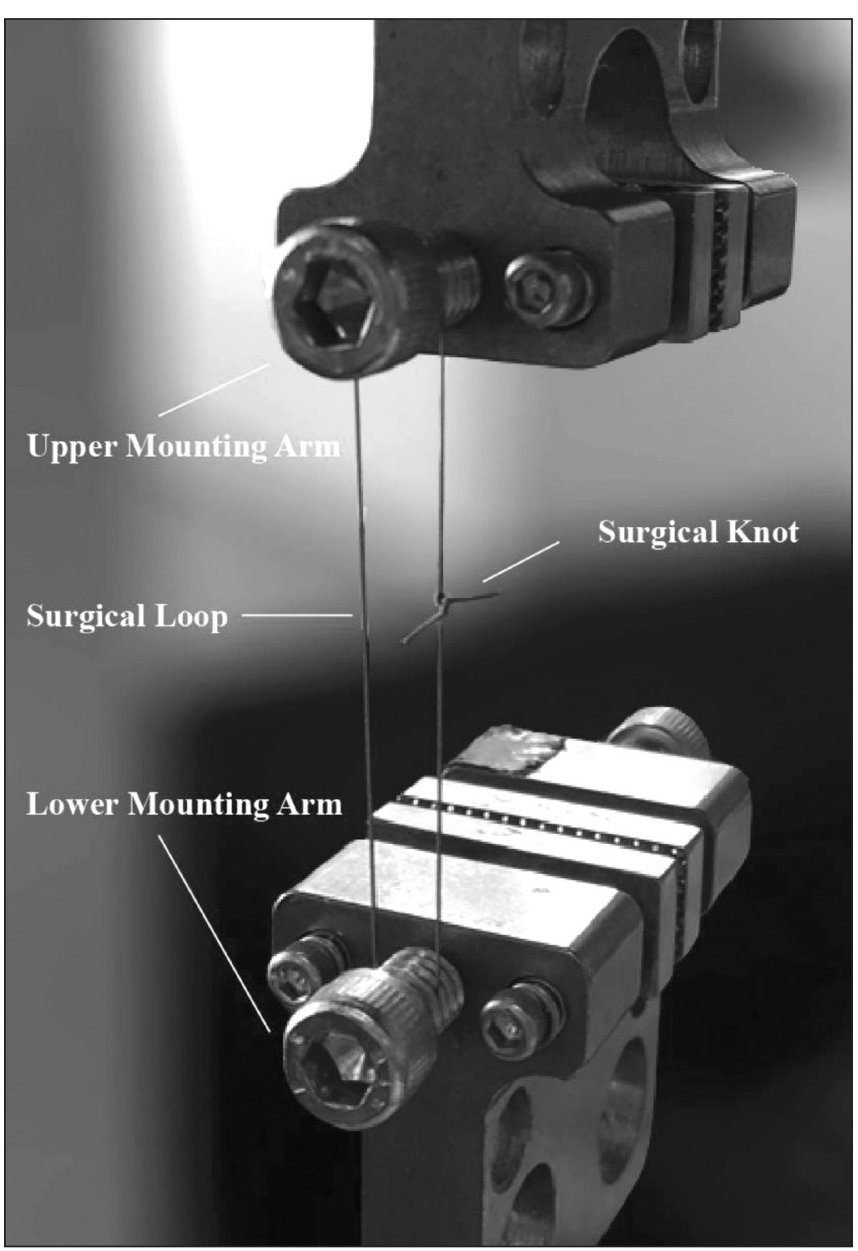

Fig. 1. Experimental setup.

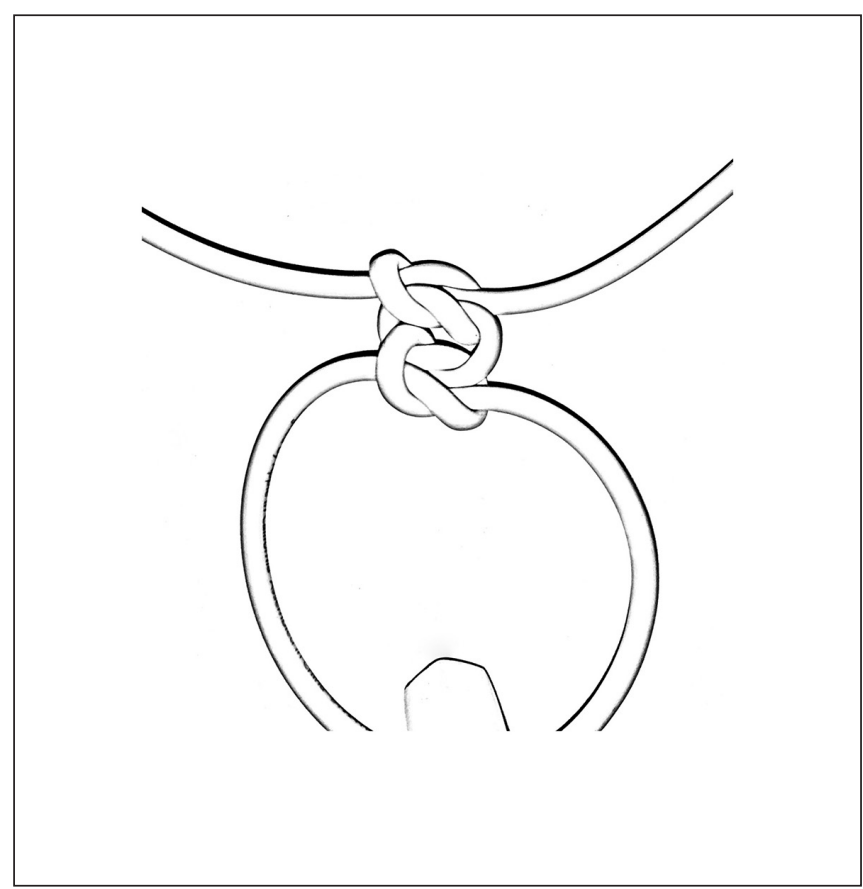

Fig. 2. Four-throw square knot configuration. 
Second, tension on the suture strands alternates between the left and right hand, producing alternating standing posts for each consecutive throw. ${ }^{6}$ Suture loops were constructed using the RHAP knot configuration composed of 4-6 throws. As it was difficult to repeatedly tie a nonslip knot with 3 throws in the RHAP configuration, the knot was modified to include an additional same-sided hitch throw as the second throw, with subsequent alternating half-hitches reversing each time. Using the knot nomenclature, the configuration of a 5-throw RHAP knot was $\mathrm{S}=\mathrm{S} / / \times \mathrm{S} / / \times \mathrm{S} / / \times \mathrm{S}$ (Fig. 3E). ${ }^{15}$ The step-by-step construction of the 5-throw RHAP knot is shown in Figure 3A-E. Similar to the square knot tests, we conducted 10 trials for each suture material and throw number to study RHAP knot strengths. To allow for direct comparison to square knots, RHAP surgical loop mounting and tensile force measurements were conducted identically to the conditions used in the square knot tests.

\section{Statistical analysis}

We performed all statistical analyses using SPSS software version 21 (IBM). We considered results to be significant at $p<0.05$. Data were initially analyzed with simple descriptive statistics, including frequencies and percentages for categorical data and means and standard deviations for tensile strength. Tensile strength was also plotted to assess the underlying distribution. Within each knot type, we compared different numbers of throws within each type of material for tensile strength using 1-way analysis of variance (ANOVA) and Tukey post hoc tests adjusted for multiple comparisons. For both silk and polyglactin 910 sutures, we compared RHAP knots against square knots for tensile strength using 1-way ANOVA with Tukey post hoc testing to reveal betweengroup differences. Finally, we used Fisher exact tests to assess suture material and whether they were prone to slippage or breakage, and we used $t$ tests to compare the difference in tensile strength at the point of knot failure between slippage and breakage. Although the tensile strength was normally distributed overall, we used box plots to illustrate the tensile strength of the material $/ \mathrm{knot}$ combinations, as this provides a better visualization of the data than simple histograms.

\section{Results}

Baseline testing with silk (Fig. 4A) and polyglactin 910 sutures (Fig. 4B) revealed that the mean tensile breaking strengths of the suture material alone were $34 \pm 3.0 \mathrm{~N}$ and $61 \pm 3.0 \mathrm{~N}$, respectively.

\section{Square knot testing}

The mean tensile strength of silk sutures for square knots in the 3-throw configuration $(24 \pm 3.2 \mathrm{~N})$ was significantly weaker than the 4-throw $(30 \pm 1.5 \mathrm{~N}, p<0.001)$ or 5-throw $(30 \pm 1.1 \mathrm{~N}, p<0.001)$ configurations. Interestingly, exceeding 4 throws did not increase the tensile

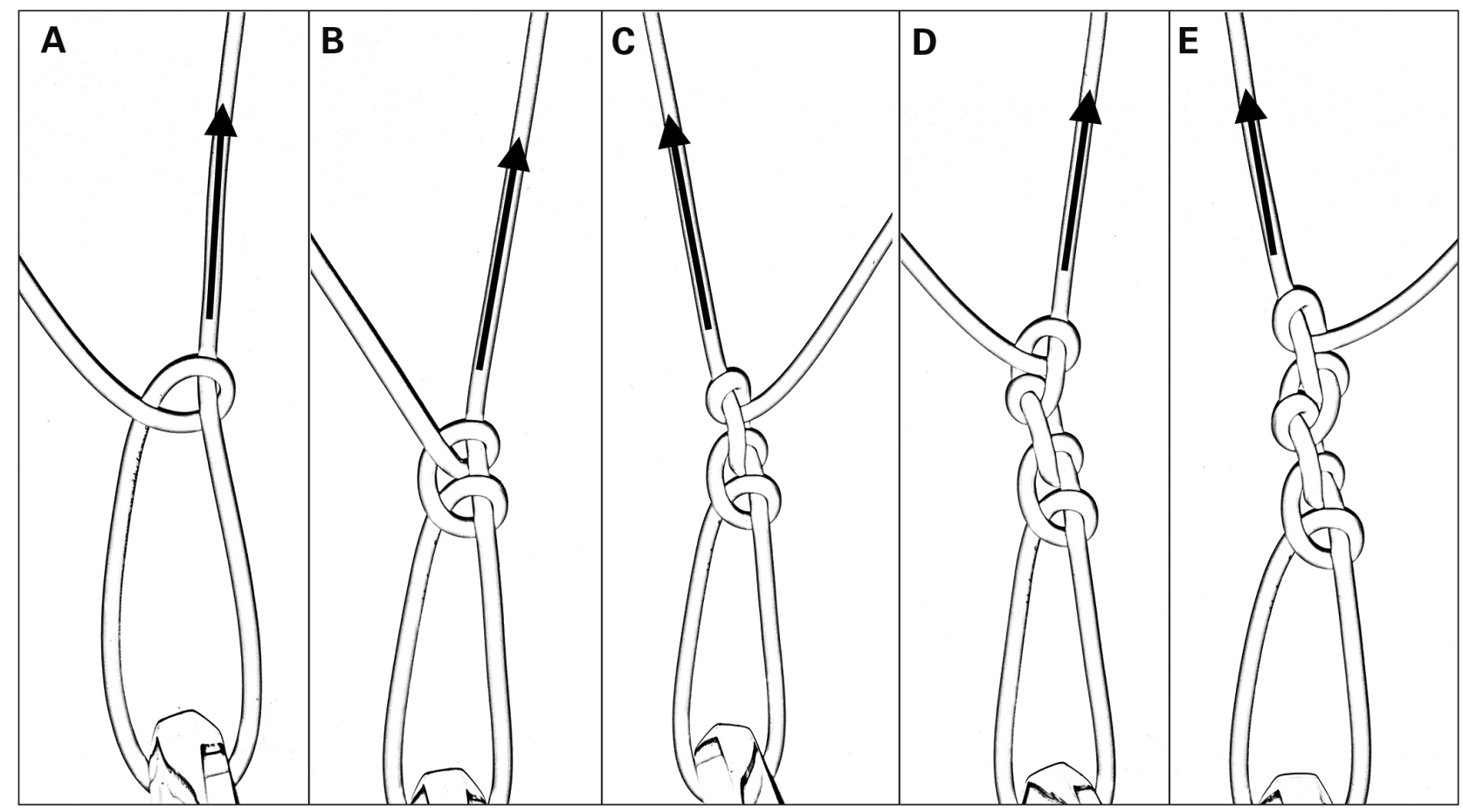

Fig. 3. Schematic for construction of reversing half-hitch alternating post (RHAP) knot, 5-throw configuration. The arrow depicts standing post. A) 1-throw, B) 2-throw same post, C) 3-throw alternating post, D) 4-throw alternating post, E) 5-throw alternating post. 
strength of the silk square knot $(p>0.99)$. Similarly, the mean tensile strength of polyglactin 910 sutures for square knots in the 3 -throw configuration $(29 \pm 8.5 \mathrm{~N})$ was significantly weaker than the 4-throw $(39 \pm 12 \mathrm{~N}, p=0.048)$ or 5-throw $(45 \pm 5.6 \mathrm{~N}, p=0.001)$ configurations. Again, with polyglactin 910 sutures, the 4-throw square knot configuration was statistically as strong as the 5-throw configuration $(p=0.29)$.

\section{RHAP knot testing}

Examination of RHAP knots in silk sutures revealed that the 4-throw configuration had a mean tensile failure strength of $23 \pm 3.4 \mathrm{~N}$, whereas 5 - and 6-throw configurations both failed at a mean tensile strength of $31 \pm$ $1.5 \mathrm{~N}$ and $31 \pm 1.2 \mathrm{~N}$, respectively. The 4-throw RHAP knot was significantly weaker than the 5 -throw $(p<$ $0.001)$ and the 6 -throw $(p<0.001)$ configurations. Maximal tensile strength in silk sutures was reached with the 5 -throw configuration; the 5-throw configuration did not differ significantly from the 6-throw configuration $(p>0.99)$. Similarly, with polyglactin 910 sutures, 4-throw knots failed at a mean tensile strength of $23 \pm$ $15 \mathrm{~N}$, which was significantly weaker than the tensions exhibited with 5 -throw $(41 \pm 13 \mathrm{~N}, p=0.013)$ and 6-throw ( $45 \pm 12 \mathrm{~N}, p=0.002)$ configurations. Knots in the RHAP configuration with polyglactin 910 sutures also reached maximal strength with the 5-throw configuration $(p=0.72)$.

\section{Square versus RHAP knots}

When examining the tensile strength of square versus RHAP knots using silk sutures (Fig. 4A), we found no significant difference in mean tensile strength between the 3 -throw square and the 4-throw RHAP knot $(p=0.64)$, between the 4-throw square and 5-throw RHAP $\operatorname{knot}(p=$ $0.93)$, or between the 5-throw square and 6-throw RHAP knot $(p=0.96)$ configurations. These results were mirrored for polyglactin 910 sutures (Fig. 4B). A summary of the comparisons can be found in Table 1 .

\section{Mechanism of failure}

Direct comparison of the suture material types demonstrated that polyglactin 910 knots, in both the square and

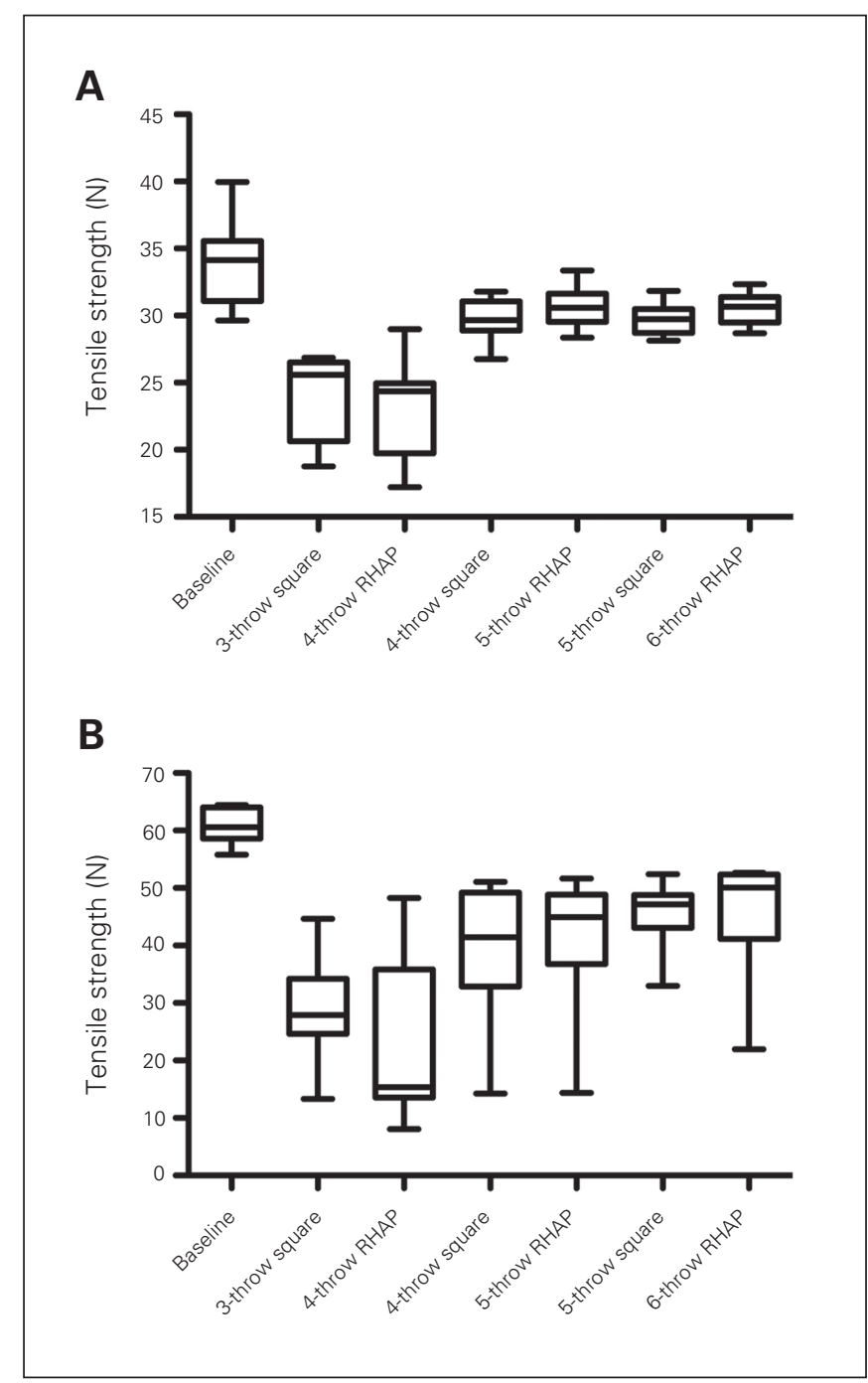

Fig. 4. Tensile strength of square knots versus reversing half-hitch alternating post (RHAP) knots in A) silk and B) polyglactin 910 sutures. Box $=25$ th and 75th percentiles, whiskers $=\min$ and $\max$ values.

\begin{tabular}{|c|c|c|c|c|c|}
\hline \multirow[b]{2}{*}{ Suture material } & \multicolumn{2}{|c|}{ Square knot } & \multicolumn{2}{|c|}{ RHAP knot } & \multirow[b]{2}{*}{$p$ value* } \\
\hline & Configuration & $\begin{array}{l}\text { Breaking strength, } \\
\text { mean } \pm \mathrm{SD}, \mathrm{N}\end{array}$ & Configuration & $\begin{array}{l}\text { Breaking strength, } \\
\text { mean } \pm \mathrm{SD}, \mathrm{N}\end{array}$ & \\
\hline \multirow[t]{3}{*}{ Silk } & 3-throw & $24 \pm 3.2$ & 4-throw & $23 \pm 3.4$ & 0.64 \\
\hline & 4-throw & $30 \pm 1.5$ & 5-throw & $31 \pm 1.5$ & 0.93 \\
\hline & 5-throw & $30 \pm 1.1$ & 6-throw & $31 \pm 1.2$ & 0.96 \\
\hline \multirow[t]{3}{*}{ Polyglactin 910} & 3-throw & $29 \pm 8.5$ & 4-throw & $23 \pm 15$ & 0.81 \\
\hline & 4-throw & $39 \pm 12$ & 5-throw & $41 \pm 13$ & $>0.99$ \\
\hline & 5-throw & $45 \pm 5.6$ & 6-throw & $45 \pm 12$ & $>0.99$ \\
\hline
\end{tabular}


RHAP configurations, were more prone to failure by slippage than silk knots $(p<0.001)$. Failure by slippage was also achieved at a lower tension than failure by breakage for both silk $(p<0.001)$ and polyglactin 910 sutures $(p<0.001)$. However, knot slippage decreased as the number of throws per knot increased, independent of knot type. A detailed breakdown of mechanism of failure can be found in Table 2.

\section{Discussion}

Suture failure can negatively impact surgical outcomes, causing serious complications postoperatively. The present study reaffirmed the current literature that suture strength varies depending on the material. ${ }^{16}$ Silk sutures were weaker than polyglactin 910 sutures at baseline and when comparing across the same knot configuration. Suture material also played a role in determining the mechanism of failure. We noted that polyglactin 910 was a stronger braided suture than silk; however, polyglactin 910 was more prone to slippage than silk within the same knot configuration. Even at the highest number of tested knot throws (5-throw square and 6-throw RHAP) slippage still occurred. This is an important takeaway from our study, as surgeons need to recognize that the inherent differences in suture material may contribute differently to the mechanism of knot failure. Additional throws may be required when using polyglactin 910 sutures to completely resist slippage.

In addition, we observed that all knotted sutures failed at the point of the knot, not elsewhere on the suture. In accordance with previous studies performed on knot mechanics, our study adds further evidence that the introduction of a knot can decrease the tensile strength of suture material. ${ }^{4}$ Looking at the mechanism of failure, the difference in mean tensile breaking strength across the different knot configurations could be attributed to the proportion of knot slippage versus breakage. As noted in the results section, knots produced with fewer throws were more prone to slippage than breakage. The introduction of additional throws in a knot increases the contact surface area for the suture

\begin{tabular}{|c|c|c|}
\hline Knot configuration & $\begin{array}{l}\text { Silk, \% failure by } \\
\text { slippage }\end{array}$ & $\begin{array}{l}\text { Polyglactin } 910, \% \\
\text { failure by slippage }\end{array}$ \\
\hline \multicolumn{3}{|l|}{ Square } \\
\hline 3-throw & 20 & 90 \\
\hline 4-throw & 0 & 40 \\
\hline 5-throw & 0 & 20 \\
\hline \multicolumn{3}{|l|}{ RHAP } \\
\hline 4-throw & 50 & 80 \\
\hline 5-throw & 0 & 30 \\
\hline 6-throw & 0 & 20 \\
\hline Total & 12 & 47 \\
\hline
\end{tabular}

material, in turn increasing the amount of friction that the suture must overcome during motion. Knots with fewer throws would accordingly have less friction, allowing them to unravel at a submaximal tensile force. With subsequent throws, additional contact points are added to the knot, increasing the friction within the knot. We noted that at higher throws (4-throw square knot and 5-throw RHAP knot) the frictional force created by the additional throws exceeded the strength of the suture at the knot, thus transitioning the mechanism of failure from slippage to breakage. It is important to note that additional throws above this transition point did not yield any additional strength.

\section{Limitations}

Monofilament sutures (3-0 gauge prolene and PDS-II) were also tested in the present study. However, monofilaments were more prone to plastic deformation under loading by the static pull machine and subsequently did not experience knot failure by breakage or slippage. One of the limitations of the static pull machine was its $10 \mathrm{~mm}$ range of travel, which precluded the testing of prolene and PDS-II sutures. As such, these materials were not included in the final analysis.

Although our study was comprehensive for polyglactin 910 and silk sutures, future studies should assess RHAP and square knot performance in monofilament sutures to enhance the generalizability of the presented data. Moreover, future studies should explore the ease and reproducibility of RHAP versus square knots within deep body cavities. If such work can be completed, RHAP may be recommended as a suitable alternative to the square knot for free-hand knot tying within deep body cavities, thereby decreasing knot failure and its associated complications.

\section{Conclusion}

Our study highlighted that the RHAP knot, with 1 additional throw, was a noninferior alternative to the square knot with silk and polyglactin 910 sutures. Furthermore, our study reaffirmed that the mechanism of failure depends on suture material, that knot breakage occurred at the knot, and that knot slippage failed with less force than knot breakage.

\begin{abstract}
Affiliations: From the School of Medicine, Faculty of Health Sciences, Queen's University, Kingston, Ont. (Wu, Sykes); the Department of Surgery, Queen's University, Kingston, Ont. (Mercer, Tang); the Clinical Research Centre, Kingston General Hospital, Kingston, Ont. (Hopman); and the Department of Public Health Sciences, Queen's University, Kingston, Ont. (Hopman).
\end{abstract}

Competing interests: None declared.

Contributors: V. Wu, D. Mercer and E. Tang designed the study. $\mathrm{V}$. Wu and E. Sykes acquired the data, which all authors analyzed. V. Wu, E. Sykes and E. Tang wrote the article, which all authors reviewed and approved for publication. 


\section{References}

1. Burkhart SS, Wirth MA, Simonich M, et al. Knot security in simple sliding knots and its relationship to rotator cuff repair: How secure must the knot be? Arthroscopy 2000;16:202-7.

2. Lo IK, Ochoa E Jr, Burkhart SS. A comparison of knot security and loop security in arthroscopic knots tied with newer high strength suture materials. Arthroscopy 2010;26(suppl):S120-6.

3. Zhao C, Hsu C-C, Moriya T, et al. Beyond the square knot: a novel knotting technique for surgical use. 7 Bone foint Surg Am 2013;95:1020-7.

4. Thacker JG, Rodeheaver GT, Moore JW, et al. Mechanical performance of surgical sutures. Am 7 Surg 1975;130:374-80.

5. Edlich RF, Long WB. Surgical knot tying manual. 3rd ed. Norwalk (CT): Covidien; 2008.

6. Dunn DL. Wound closure manual. Somerville (NJ): Ethicon Inc; 2007.

7. Graumont R, Hensel J. Encyclopedia of knots and fancy rope work. 4th ed. Atglen (PA):Schiffer; 1952.

8. Behm T, Unger JB, Ivy JJ, et al. Flat square knots: Are 3 throws enough? Am 7 Obstet Gynecol 2007;197:172e1-3.
9. Nottage WM, Lieurance RK. Arthroscopic knot tying techniques. Arthroscopy 1999;15:515-21.

10. van Rijssel EJ, Trimbos JB, Booster MH. Mechanical performance of square knots and sliding knots in surgery: comparative study. $A m \mathcal{F}$ Obstet Gynecol 1990;162:93-7.

11. Gunderson PE. The half-hitch knot: a rational alternative to the square knot. Am f Surg 1987;154:538-40.

12. Trimbos JB. Security of various knots commonly used in surgical practice. Obstet Gynecol 1984;64:274-80.

13. Mahar AT, Moezzi DM, Serra-Hsu F, et al. Comparison and performance characteristics of 3 different knots when tied with 2 suture materials used for shoulder arthroscopy. Arthroscopy 2006;22: 614.e1-2.

14. Muffly TM, Boyce J, Kieweg SL, et al. Tensile strength of a surgeon's or a square knot. 7 Surg Educ 2010;67:222-6.

15. Loutzenheiser TD, Harryman DT, Yung SW, et al. Optimizing arthroscopic knots. Arthroscopy 1995;11:199-206.

16. Marturello DM, McFadden MS, Bennett RA, et al. Knot security and tensile strength ofsuture materials. Vet Surg 2014;43:73-9.

\section{Canadian Journal We believe in open access to research} of Surgery

To ensure continued worldwide free access to all CFS content, articles submitted for publication as of Jan. 1, 2014, are subject to a submission fee of $\$ 100$ (Canadian funds). Submission fees will be waived for corresponding authors affiliated with $C 7 S$ sponsors. Accepted Research, Review and Continuing Medical Education articles are subject to a publication fee of $\$ 700$, and Commentaries and Discussions are subject to a publication fee of $\$ 500$, payable on acceptance in Canadian funds.

\section{Benefits of open access}

- For researchers and institutions: increased visibility, usage and impact for their work

- For government: a better return on investment for funding research

- For society: efficient, effective patient care resulting in better outcomes

CFS articles are available free of charge on the journal website (canjsurg.ca) and in PubMed Central. 